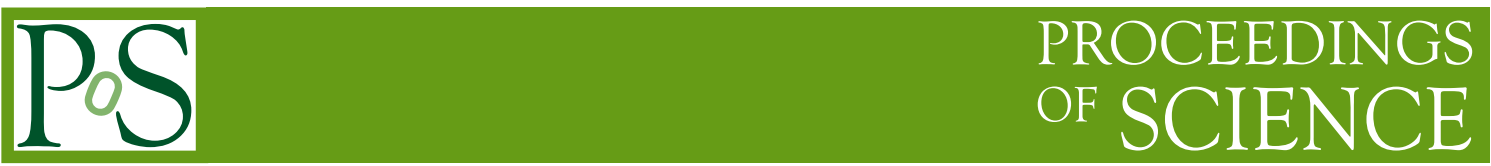

\title{
Neutrinos from Stored Muons
}

\section{K. Long*}

Imperial College London and STFC

E-mail: K.Long@Imperial.AC.UK

\begin{abstract}
The nuSTORM facility will provide $v_{e}\left(\bar{v}_{e}\right)$ and $v_{\mu}\left(\bar{v}_{\mu}\right)$ beams from the decay of low energy muons confined within a storage ring. The instrumentation of the ring, combined with the excellent knowledge of muon decay, will make it possible to determine the neutrino flux at the \%-level or better. The neutrino and anti-neutrino event rates are such that the nuSTORM facility, serving a suite of near detectors, will allow measurements of the $v_{e} A\left(\bar{v}_{e} A\right)$ and $v_{\mu} A\left(\bar{v}_{\mu} A\right)$ cross sections to be made with the precision required to enhance the sensitivity of the next generation of longbaseline neutrino-oscillation experiments thereby enhancing their discovery potential. By delivering precise cross section measurements with a pure weak probe nuSTORM has the potential to make measurements important to understanding the physics of nuclei. The precise knowledge of the initial neutrino flux also makes it possible to deliver uniquely sensitive light sterile-neutrino searches. The concept for the nuSTORM facility will be presented together with an evaluation of its performance. The status of the planned consideration of nuSTORM at CERN in the context of the Physics Beyond Colliders Study Group will be summarised.
\end{abstract}

XVII International Workshop on Neutrino Telescopes

13-17 March 2017

Venezia, Italy

${ }^{*}$ Speaker. 


\section{Introduction}

Intense muon beams of low emittance have the potential to serve a programme of neutrino physics that includes percent-level-precision measurements of electron- and muon-neutrino-nucleus scattering, exquisitely sensitive searches for sterile neutrinos and detailed studies of neutrino oscillations. A "neutrino factory", in which a muon beam circulates within a storage ring, the longstraight sections of which point to distant neutrino detectors, generates large, equal, fluxes of electron- and muon-neutrinos $[1,2,3,4,5,6]$. The charge-to-mass ratio of the muon makes it possible to optimise a neutrino factory such that the neutrino-beam energy is matched to a particular choice of detector technology and source-detector distance [7, 8]. The mass of the muon, $\sim 200$ times that of the electron, suppresses bremsstrahlung and beamsstrahlung by a factor of $\sim 10^{5}$ below that which would pertain for electron or positron beams. This makes it possible to conceive of a "muon collider" $[9,10]$, which may provide the only cost-effective means to deliver lepton-anti-lepton collisions at centre-of-mass energies above 1.5 TeV [11].

nuSTORM ("Neutrinos from Stored Muons") is a facility based on a low-energy muon decay ring (see figure 1) [12,13]. Pions, produced in the bombardment of a target, are captured in a magnetic channel. The magnetic channel is designed to deliver a pion beam with central energy $E_{\pi}$ and energy spread $\sim \pm 20 \% E_{\pi}$ to the muon decay ring. The pion beam is injected into the production straight of the decay ring. Roughly half of the pions decay as the beam passes through the production straight. At the end of the straight, the return arc selects a muon beam of central energy $E_{\mu}<E_{\pi}$ and energy spread $\sim \pm 10 \% E_{\mu}$ that then circulates. Undecayed pions are directed to a beam dump. A detector placed on the axis of the production straight will receive a bright flash of muon neutrinos from pion decay followed by a series of pulses of muon and electron neutrinos from subsequent turns of the muon beam. Appropriate instrumentation in the decay ring and production straight will be capable of determining the integrated neutrino flux with a precision of $\lesssim 1 \%$ [13]. The flavour composition of the neutrino beam from muon decay is known and the neutrino-energy spectrum can be calculated precisely using the Michel parameters and the optics of the muon decay ring. The pion and muon energies $\left(E_{\pi}\right.$ and $\left.E_{\mu}\right)$ can be optimised to:

- Measure $v_{e} A\left(\bar{v}_{e} A\right)$ and $v_{\mu} A\left(\bar{v}_{\mu} A\right)$ interactions with per-cent-level precision; and

- Search for sterile neutrinos with exquisite sensitivity.

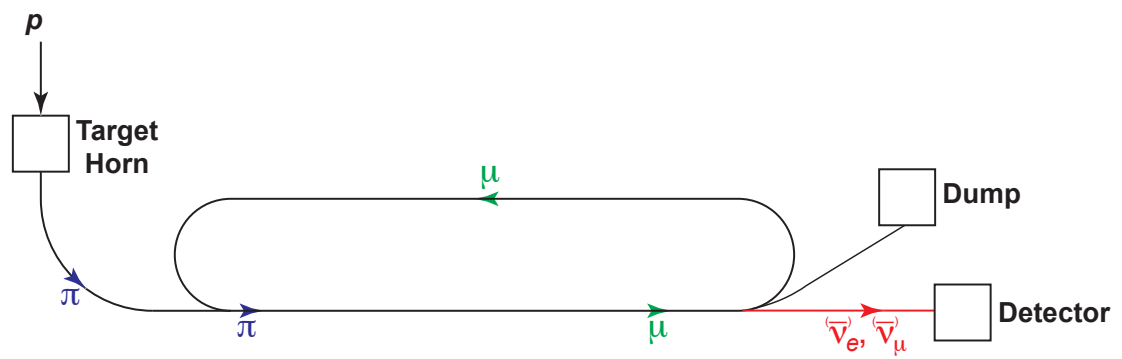

Figure 1: Schematic of the nuSTORM neutrino-beam facility. 
A muon beam with an energy of $3.8 \mathrm{GeV}$, derived from an injected pion beam of energy $5 \mathrm{GeV}$, was proposed in [13] to search for sterile neutrinos using a magnetised detector placed $1.8 \mathrm{~km}$ from the end of the production straight. With $10^{21}$ protons on target, the proposed configuration was shown to be able to test the LSND [14] and MiniBooNE [15, 16] anomalies with $10 \sigma$ sensitivity [17]. The case for a neutrino-scattering programme was also outlined in [13]. This contribution to the Neutrino Telescopes workshop presents the case for nuSTORM as a facility at which a definitive neutrino-nucleus-scattering programme can be carried out that will be ground-breaking in its precision and the characterisation of the final state. A brief review of the importance of the study of neutrino interactions and the benefits that precise knowledge of the neutrino flux will bring is presented in section 2. Section 3 summarises the status of the evaluation of the nuSTORM accelerator complex. The benefits of nuSTORM and the work being undertaken in the context of the CERN Physics Beyond Colliders Study Group [18] is summarised in section 4. Finally, conclusions are drawn in section 5 .

\section{Neutrino-nucleus scattering}

\section{Impact on searches for leptonic CP-invariance violation}

The search for CP-invariance violation (CPiV) in present and planned long-baseline neutrinooscillation experiments relies on the measurement of the rate of $v_{e}\left(\bar{v}_{e}\right)$ appearance in $v_{\mu}\left(\bar{v}_{\mu}\right)$ beams. The phenomenological description of the effect relies on the assumption of three neutrinomass eigenstates that mix to produce the three neutrino flavours [19, 20, 21, 22]. CPiV arises in this framework if the value of a phase parameter, $\delta$, is such that $\sin \delta \neq 0$.

The oscillation probability is a function of the source-detector distance (the baseline) and the neutrino energy. Typical baselines range from $295 \mathrm{~km}$ for T2K [23] and the proposed Hyper-K experiment [24, 25, 26, 27], $800 \mathrm{~km}$ for NOvA [28] and $1300 \mathrm{~km}$ for the DUNE experiment [29, $30,31,32]$. The (anti)neutrino beam interacts with the material in the earth as it propagates from source to detector. The elastic interaction of electron neutrinos with atomic electrons includes a charge-exchange contribution that is absent from the interactions of anti-electron neutrinos. This introduces a "matter effect" and causes the oscillation probability of electron neutrinos to differ from that of anti-electron neutrinos, introducing an "apparent" CPiV effect that depends on the neutrino mass hierarchy. The discovery of $\mathrm{CPiV}$ in neutrino oscillations requires that the "true" $\mathrm{CPiV}$ that depends on $\delta$ be distinguished from the apparent $\mathrm{CPiV}$ that arises from neutrino interactions with the earth.

The "CP asymmetry", $A_{\mathrm{CP}}$, given by:

$$
A_{\mathrm{CP}}=\frac{P\left(v_{\mu} \rightarrow v_{e}\right)-P\left(\bar{v}_{\mu} \rightarrow \bar{v}_{e}\right)}{P\left(v_{\mu} \rightarrow v_{e}\right)+P\left(\bar{v}_{\mu} \rightarrow \bar{v}_{e}\right)}
$$

where $P\left(v_{\alpha} \rightarrow v_{\beta}\right)$ is the probability for the transition $v_{\alpha} \rightarrow v_{\beta}$, may be used to study the size the $\mathrm{CPiV}$ effect. The $\mathrm{CP}$ asymmetry is shown as a function of baseline in figure 2 . The matter-induced $\mathrm{CP}$ asymmetry grows from $\sim 10 \%$ at $295 \mathrm{~km}$ to $\sim 40 \%$ at $1300 \mathrm{~km}$. The "true" CPiV effect is maximised when $\delta= \pm \frac{\pi}{2}$. For maximal CPiV, the true CP asymmetry is $\sim 38 \%$ at $295 \mathrm{~km}, \sim 35 \%$ at $800 \mathrm{~km}$ and $\sim 30 \%$ at $1300 \mathrm{~km}$. For a long-baseline experiment to be capable of excluding CPinvariance conservation at the $3 \sigma$ confidence level for $75 \%$ of all possible values of $\delta$ requires 
that the total uncertainty on $A_{\mathrm{CP}}$ be $<5 \%$ ( $\lesssim 5 \%$ for a baseline of $295 \mathrm{~km}, \lesssim 4.5 \%$ for a baseline of $800 \mathrm{~km}$ and $\lesssim 4 \%$ for a baseline of $1300 \mathrm{~km}$ ). Taking $5 \%$ to be the requirement for the total uncertainty on a measurement of $A_{\mathrm{CP}}$, an estimate for the systematic uncertainty may be derived by assuming that, when enough data is taken, the systematic uncertainty may be no larger than the statistical uncertainty. Under these assumptions, to establish CPiV from observations of $v_{e}$ and $\bar{v}_{e}$ appearance requires that oscillation probabilities be measured with a precision of $\lesssim 3 \%$.

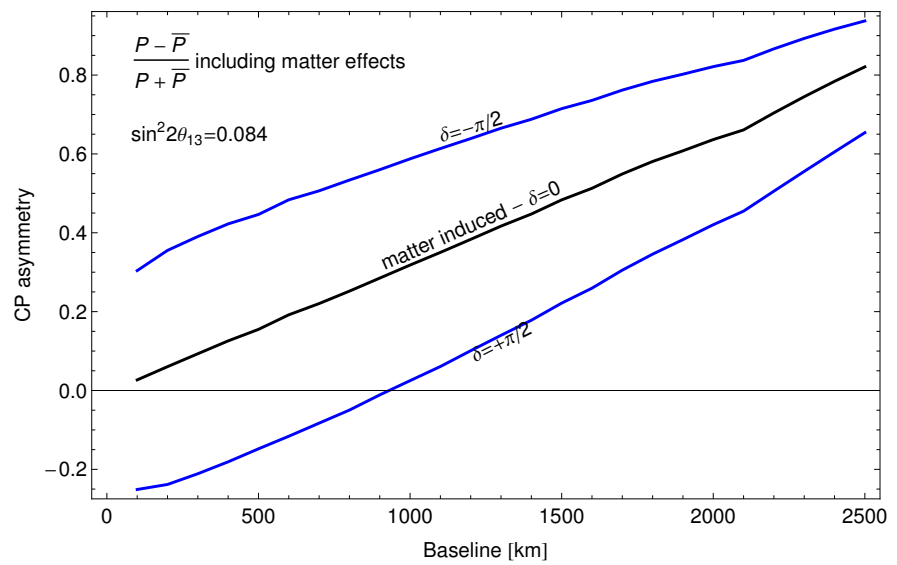

Figure 2: $\mathrm{CP}$ asymmetry (equation 2.1) plotted as a function of source-detector distance (baseline). Taken from [33]. The solid black line shows the asymmetry that arises without CP-invariance violation in oscillations (i.e., with $\delta=0$ ) due to the interactions of electron neutrinos with atomic electrons in the earth's crust. The upper, solid, blue line shows the asymmetry assuming $\delta=-\frac{\pi}{2}$, while the lower, solid, blue line shows the asymmetry assuming $\delta=\frac{\pi}{2}$.

The projected sensitivity to CPiV of the DUNE experiment is plotted as a function of exposure in figure 3 [30]. An exposure of $288 \mathrm{kt} \mathrm{MW}$ years will be achieved after seven years of running, with the planned staging to reach a total detector mass of $40 \mathrm{kt}$ detector and a proton beam-power of 1.2 MW [34]. Equal exposures in neutrino and antineutrino mode have been assumed. The DUNE collaboration presents the sensitivity as a function of the assumed normalisation uncertainties on the $v_{e}$ and $\bar{v}_{e}$ appearance signals. Reducing the $\bar{v}_{e}$ normalisation uncertainty from $3 \%$ to $1 \%$ brings the exposure required to exclude CP invariance at the $3 \sigma$ confidence level over $75 \%$ of all possible values of $\delta$ down from $\sim 1200 \mathrm{kt} \mathrm{MW}$ years to $\sim 600 \mathrm{kt} \mathrm{MW} \mathrm{years.}$

The projected sensitivity of the Hyper-K experiment, updated from [25], is also shown in figure 3. An exposure of $13 \mathrm{MW} \times 10^{7} \mathrm{~s}$ will be achieved after ten years assuming a 1:3 ratio between neutrino and anti-neutrino running. The planned staged implementation of two $187 \mathrm{kt}$ detectors is indicated, a proton beam-power of $1.3 \mathrm{MW}$ at $30 \mathrm{GeV}$ has been assumed. The systematic uncertainties assumed by the Hyper-K collaboration in their estimation of the CPiV sensitivity of their experiment are summarised in figure 4 [25]. The total systematic uncertainty is dominated by the combined "flux and near-detector" and the "cross-section model" uncertainties.

In addition to systematic uncertainties, a lack of knowledge of $v_{e, \mu} A\left(\bar{v}_{e, \mu} A\right)$ cross sections or inaccuracies in the simulation of the hadronic final state can lead to biases in the parameters extracted from the data. Such biases may arise, for example, from mis-classification of events [35] or mis-reconstruction of the energy of the incident neutrino [36, 37, 38, 39]. A discussion of 

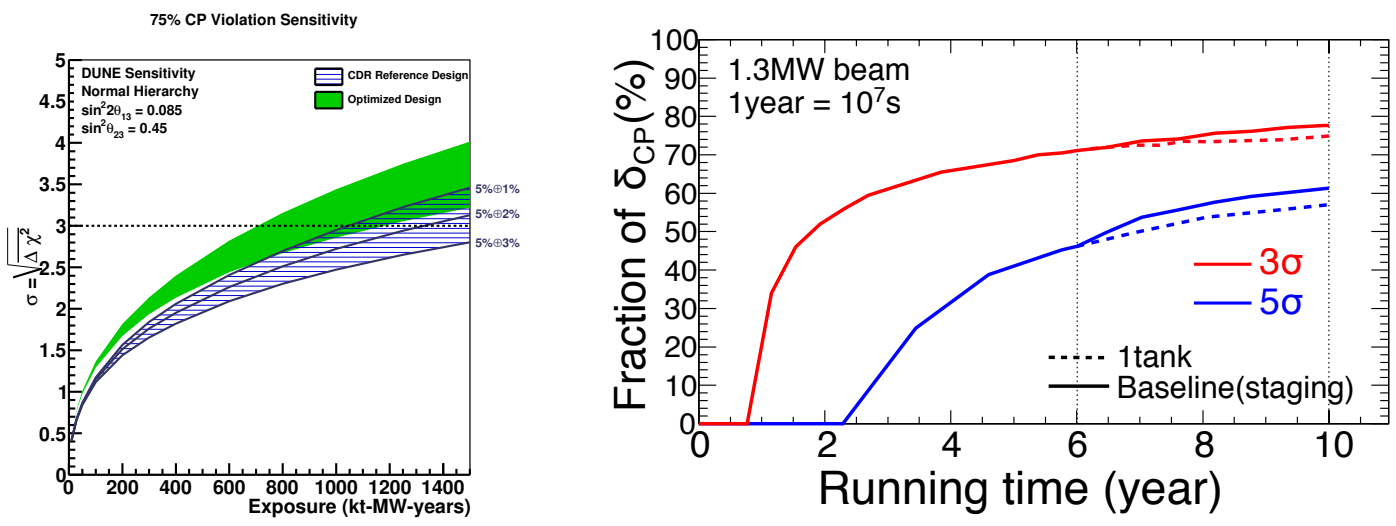

Figure 3: Left panel: Expected sensitivity of the DUNE experiment to CP-invariance violation plotted as a function of exposure in $\mathrm{kt} \cdot \mathrm{MW} \cdot \mathrm{years}$ assuming equal running in neutrino and antineutrino mode, for a range of values for the $v_{e}$ and $\bar{v}_{e}$ signal-normalisation uncertainties (from $5 \%$ to $1 \%$ ) added in quadrature to an uncertainty of $5 \%$ on the normalisation of the background. The sensitivities shown are for the exclusion of CP-invariance conservation over $75 \%$ of the available range of values of $\delta$ assuming the normal hierarchy. The two bands represent a range of potential beam designs: the blue hashed band is for the CDR Reference Design and the solid green band is for the optimised design. The figure is taken from [30]. Right panel: fraction of all values of the $\mathrm{CPiV}$ phase, $\delta\left(=\delta_{\mathrm{CP}}\right)$, for which $\delta=\delta_{\mathrm{CP}}=0, \pi$ can be excluded at $3 \sigma$ (red) or $5 \sigma$ (blue) plotted as a function of running time. An exposure of $13 \mathrm{MW} \times 10^{7} \mathrm{~s}$ is expected to be achieved after 10 years of operation. Figure updated from [25].
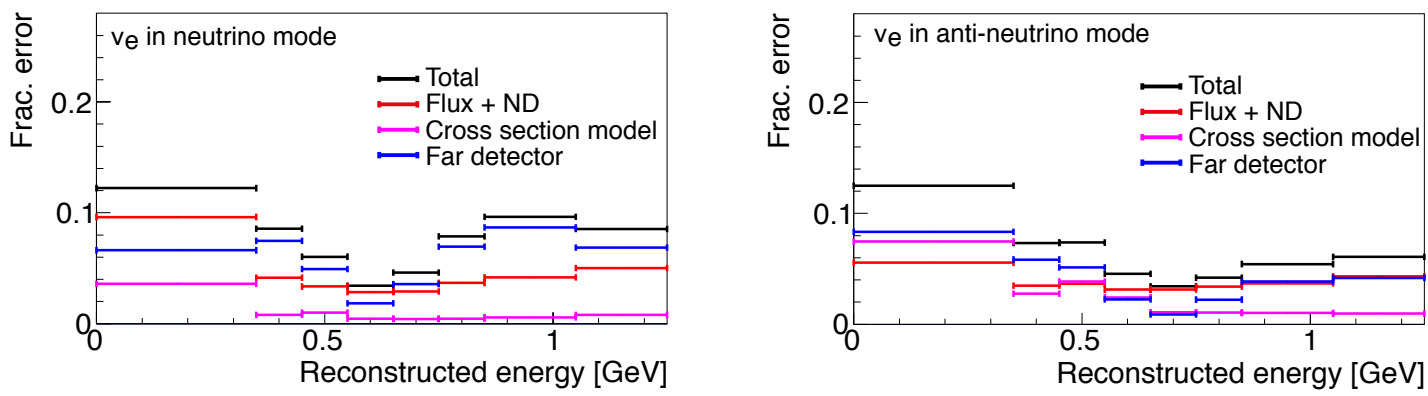

Figure 4: Fractional uncertainty for $v_{e}$ (left panel) and $\bar{v}_{e}$ (right panel) appearance. The solid black points show the total uncertainty, the red points show the flux and cross-section uncertainty constrained by the near detector measurements, the magenta points show the near detector non-constrained cross-section uncertainty, the blue points show the far detector uncertainty. Figures taken from from [25].

possible sources of bias is presented in [33]. When searching for CPiV, any effects that differ in $v_{e} A$ and $\bar{v}_{e} A$ scattering and are not quantitatively understood are particularly pernicious since such a difference may be mis-interpretted as a signal for $\mathrm{CPiV}$.

The next generation of long-baseline neutrino-oscillation experiments, DUNE and Hyper-K have the potential to observe $\mathrm{CPiV}$ violation. To maximise the scientific impact of the large, exquisitely-precise data sets that they will collect requires that the $v_{e}\left(\bar{v}_{e}\right)$ and $v_{\mu}\left(\bar{v}_{\mu}\right)$ cross sections be known with percent-level precision and that uncertainties associated with cross-section models 
are also under control at the percent level.

\section{Potential for impact on understanding of the structure of the nucleus}

Theoretical understanding of the structure of the nucleon is detailed and precise and can be used to predict cross sections for a wide variety of processes over a wide kinematic range. However, a number of measurements, such as the spin structure of the nucleon, challenge the present understanding [40]. The theoretical description of the structure of the nucleus is not quite as accurate and requires development, for example to describe correlations among the nucleons that make up the nucleus [41]. Phenomenological models of lepton-nucleus scattering are based on the present understanding of nuclear physics and exploit a wealth of data to determine a number of phenomenological parameters. Such models have been shown to give a reasonable description of some of the present neutrino-nucleus scattering data but may fail when used to extrapolate beyond the range of energies, nuclei, or types of process on which they have been "tuned" [42]. A review of the challenges that must be overcome to deliver a good description of the hadronic final states is presented in [43].

A vibrant experimental programme is underway to extend and improve the scattering database on which the theoretical and phenomenological description of the nucleus relies. In the case of electron-nucleus scattering, the $12 \mathrm{GeV}$ upgrade to the CEBAF accelerator at Jefferson Laboratory combined with the new experimental facilities and detector upgrades will provide a wealth of new data that will, most likely, lead to new insights (see for example [44]). Neutrino-nucleus scattering has made both seminal and historic contributions to the development of nuclear structure. The neutrino offers a probe that is polarised and is sensitive to flavour and isospin. It is conceivable that neutrino-nucleus scattering has a role to play in unravelling issues such as the orbital contribution to the spin of the nucleon and the nature of nucleon-nucleon correlations. A facility that is able to deliver a precisely calibrated flux is required if neutrino-nucleus scattering measurements are to contribute to the understanding of nuclear structure.

\section{3. nuSTORM; the facility}

A detailed study of nuSTORM that includes consideration of the implementation of the facility at FNAL may be found in [13, 45, 46]. A preliminary investigation of possible options for siting the facility at CERN that used the FNAL study as the basis may be found in [47, 48]. In these studies the facility was optimised for the search for a light sterile neutrino. A short description of the principal elements of the nuSTORM facility will be given here together with a summary of the considerations that will inform the re-optimisation of the facility for the study of neutrino-nucleus scattering.

A schematic drawing of the layout is shown in figure 5 [45]. Pions are produced when a proton beam of energy $\sim 100 \mathrm{GeV}$ strikes a solid target. In the FNAL study, it was assumed that one booster batch per cycle would be directed from the Main Injector to the nuSTORM target. At CERN the facility could be served using fast extraction from the SPS. The target is inserted into the neck of the horn (see figure 6) [49]. Using a genetic algorithm, the geometry of the target and horn has been optimised to focus pions into a quadrupole channel that transports the sign-selected 
pion beam to the decay ring. A chicane in the transport channel selects the momentum of the pion beam that will be injected into the ring [50].
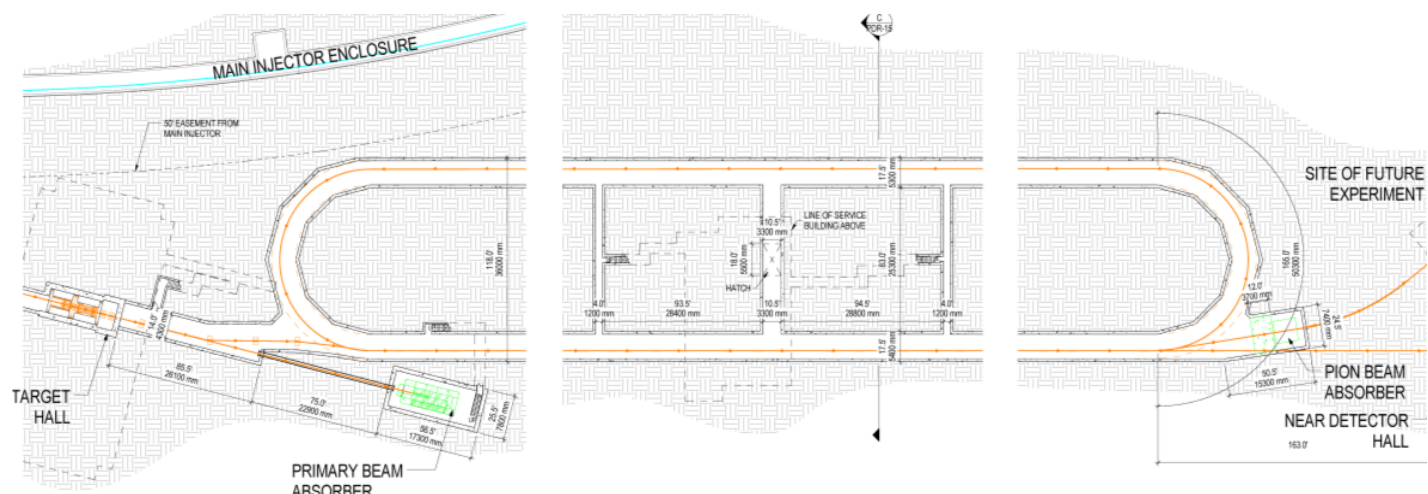

Figure 5: Plan view of the nuSTORM decay-ring enclosure as laid out on the FNAL site (taken from [45]). The target hall and beam transport to the ring is shown together with an outline of the proton-beam dump. The line by which undecayed pions and off-momentum muons are guided to the pion-absorber is also shown. The Main Injector enclosure is shown at the top of the figure.
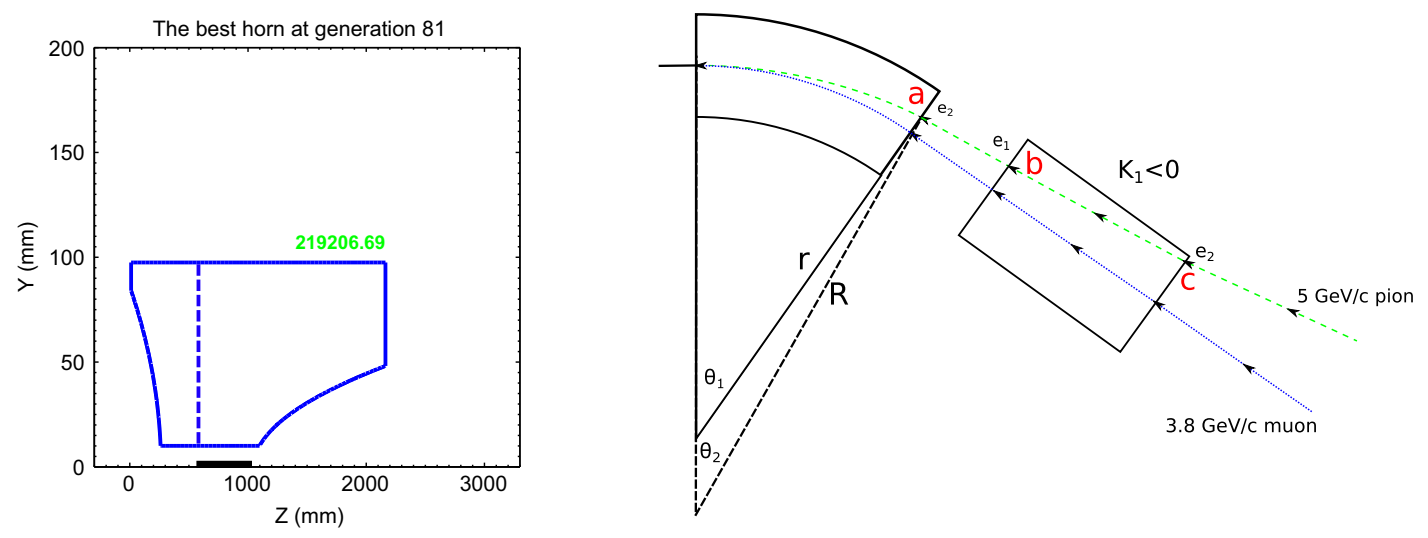

Figure 6: Left panel: The shape of the optimized horn with a $46 \mathrm{~cm}$ inconel target (taken from [49]). Right panel: Schematic drawing of the reference orbits in the orbit combination section (OCS) magnets (taken from [50]). The injected pions and circulating muons move as indicated by the arrows.

Pion injection into the production straight of the decay ring is achieved by placing the pion beam onto an appropriate trajectory at the end of the return arc where dispersion is large. The optics of the lattice then bring the pion beam onto the orbit of the circulating muon beam as the dispersion is reduced to the low value required in the production straight (see figure 6) [50]. The portion of the accelerator chain that achieves this is referred to as the orbit combination section (OCS).

Two options for the nuSTORM decay ring have been considered. The baseline is a compact, large-aperture $(60 \mathrm{~cm})$ quadrupole-focusing ring $[13,51]$. The arcs exploit double-bend achromat optics, which, in the FNAL design, were optimised to transport a beam momentum of $(3.8 \pm$ 
$10 \%) \mathrm{GeV} / \mathrm{c}$. Approximately $50 \%$ of the pions decay in the $185 \mathrm{~m}$ production straight. The length of one booster batch is $1.6 \mu \mathrm{s}$. This is well matched to the time taken for the muon beam to complete one turn in the storage ring since pion injection will stop as the muon beam re-passes the OCS. Undecayed pions and muons that do not fall within the momentum acceptance of the ring are guided to a pion absorber by a mirror of the OCS used to inject pions into the ring. A significant flux of low-momentum muons emerges from the pion absorber and can be used to serve a six-dimensional cooling experiment or other muon-beam-related $R \& D$. The alternative design for the storage ring exploits fixed-field alternating-gradient (FFAG) optics to increase the momentum acceptance of the ring $[52,53]$. Designs have been presented that are capable of accommodating a momentum byte of $\pm 20 \%$.

To realise the full potential of nuSTORM for the measurement of $v A$ scattering cross sections requires that the neutrino-flux normalisation be measured with a precision small compared to the measurement-uncertainty arising from the detection of neutrino-scattering events. The specification adopted for the storage-ring instrumentation is that the neutrino-flux normalisation shall be known with a precision of $\lesssim 1 \%$. To meet this specification requires that: the circulating-muon intensity be measured with a precision $<1 \%$; the mean momentum is determined with a precision $<1 \%$; the momentum spread is known with a precision $\leq 1 \%$; and the mean tune is known to $\sim 0.01$ [54]. The development of a coherent set of instrumentation capable of meeting these specifications is an important item for the development of nuSTORM.

The neutrino detectors described in [54] were developed to deliver a definitive sterile-neutrino search. Many groups are developing powerful detectors capable of allowing the study of exclusive final states in neutrino-nucleus scattering. Concepts such as those that are presently being considered as near detectors for DUNE [32] and Hyper-K [24, 25, 26] should be considered when developing a full proposal for nuSTORM to serve a definitive neutrino-nucleus scattering programme.

\section{4. nuSTORM and the CERN Physics Beyond Colliders study}

In September 2016 CERN established the Physics Beyond Colliders (PBC) study group to consider ways in which the accelerators at CERN could be used or extended to support a diverse physics programme to complement the energy-frontier physics being pursued using the LHC [18]. The feasibility of implementing nuSTORM at CERN was included as a work package in the PBC study. Within the PBC context, the scientific objectives of nuSTORM are:

- To make detailed and precise measurements of neutrino-nucleus interactions not only as a service to the long- and short-baseline neutrino oscillation programmes but also as a means of studying the nucleus using a weak probe and seeking evidence for non-standard interactions; and

- To take forward the search for light sterile neutrinos should the results of the Short Baseline Neutrino (SBN) programme at FNAL [55] indicate that such a programme is required.

The potential for nuSTORM to establish a new technique for the study of fundamental particles and their interactions is recognised. The PBC study does not include consideration of a possible six-dimensional-cooling experiment (see for example [56]) to follow the demonstration of the 
reduction of normalised transverse emittance that will be provided by the international Muon Ionization Cooling Experiment (MICE) [57].

Measurements of the total charged-current $v_{\mu} A\left(\bar{v}_{\mu} A\right)$ total cross section are shown as a function of reconstructed neutrino energy in figure 7 [58]. The results of phenomenological calculations are also shown. The calculations of the total cross section are consistent with the measurements. To evaluate the total cross section it is broken down into phenomenologically-convenient components; these are also shown in figure 7. For neutrino scattering, deep inelastic scattering (DIS) takes over from resonance production for neutrino energies between $4 \mathrm{GeV}$ and $5 \mathrm{GeV}$. In the case of anti-neutrino scattering, the handover from resonance production to DIS takes place between $7 \mathrm{Gev}$ and $8 \mathrm{GeV}$. Below $\sim 1 \mathrm{GeV}$, the charged-current quasi-elastic (CCQE) process dominates. The resonance-production process makes a significant contribution in the range $1 \lesssim E_{V} \lesssim 6 \mathrm{GeV}$. For neutrino energies in this range, the relative contributions of DIS and resonance production can be determined if the four-momentum transfer squared $\left(Q^{2}\right)$ and the total hadronic energy $(W)$ can be reconstructed. The partition between the various classes (DIS, resonance production and CCQE) is determined by fitting model parameters to the available data. Uncertainties in the resulting contributions to the total cross section contribute to the uncertainties and biases discussed in section 2 . Precise measurements of $v_{e, \mu} A\left(\bar{v}_{e, \mu} A\right)$ scattering are required to refine the models and to determine with precision their relative contributions.

The sensitivity of all oscillation experiments depends on the precision of models of the structure of the nucleus and the details of simulations of neutrino scattering. nuSTORM will provide a beam of precisely known flavour for which the flux will be known to $1 \%$ or better. Instrumented with a detector capable of making measurements of exclusive final states and of reconstructing $Q^{2}$ and $W$, nuSTORM can deliver the data required for precise models of the nucleus and of neutrinonucleus scattering to be developed if the neutrino-beam energy spans the range $1 \lesssim E_{v} \lesssim 6 \mathrm{GeV}$. These considerations lead to the following specification for the energy of the circulating muon beam:

- Maximum stored muon energy, $E_{\mu}=6 \mathrm{GeV}$; and

- It must be possible to vary the muon-beam energy in the range $1 \lesssim E_{\mu} \lesssim 6 \mathrm{GeV}$.

Since the neutrino-energy spectrum is precisely known once the muon-beam energy is specified, the falling edge of the neutrino-energy spectrum can be used to calibrate the energy response of the neutrino detectors. Further, by combining data taken with different stored-muon energies, as described for NuPRISM in [59], cross sections may be determined in narrow neutrino-energy bands.

The accelerator study will be split into two parts: the re-optimisation of the pion-capture section and the muon-decay ring for the neutrino-scattering programme; and the study of the feasibility of implementing the facility at CERN. The re-optimisation will start from the study of the capture of $\sim 8 \mathrm{GeV}$ pions and their transport to the decay ring. The optimisation of the OCS for the higher pion-beam energy will be considered. The design of the decay ring will be revised to accommodate the maximum muon-beam energy of $6 \mathrm{GeV}$ and to provide the ability to store muon beams with energies in the range $1 \lesssim E_{\mu} \lesssim 6 \mathrm{GeV}$. The feasibility study will develop a preliminary proposal for siting the facility at CERN and will take into account the number of protons on target required, 

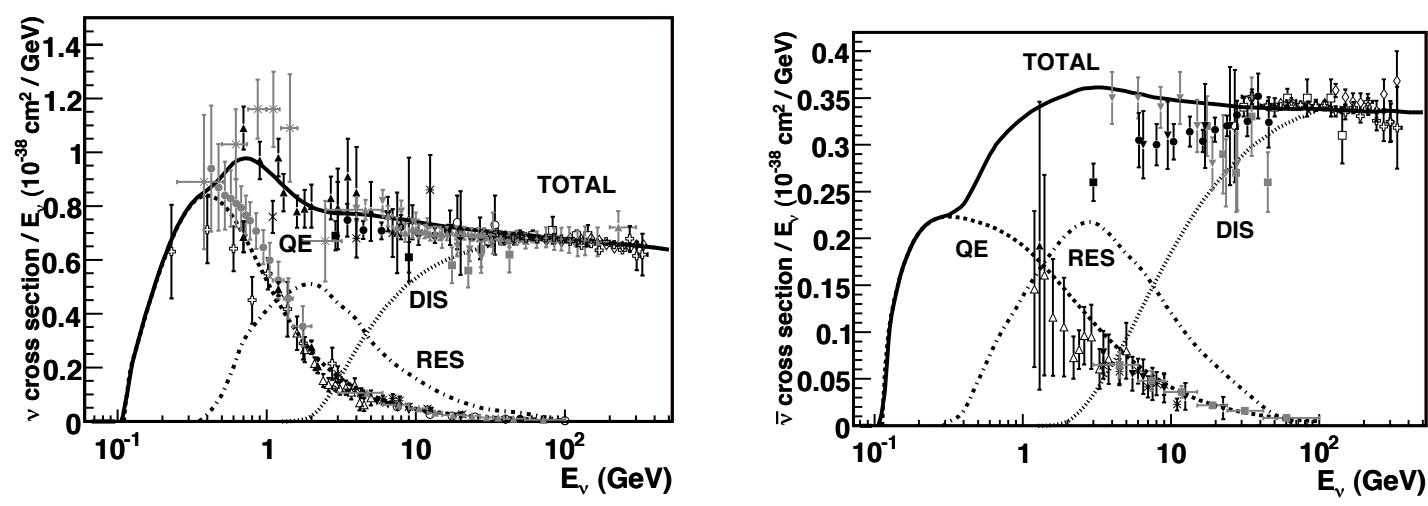

Figure 7: Total neutrino and anti-neutrino charged-current cross section per nucleon (for an isoscalar target) divided by neutrino energy and plotted as a function of neutrino energy (taken from [58]). The results of calculations of the processes into which the total cross section is broken down are also shown. These contributions include quasi-elastic scattering (dashed), resonance production (dot-dash), and deep inelastic scattering (dotted). Example predictions for each are provided by the NUANCE generator [60]. The quasielastic scattering data and predictions have been averaged over neutron and proton targets.

the extracted-beam parameters and the number of potential users. Fast extraction from the SPS and proton-beam transport to the pion-production target will be considered alongside initial engineering of the target, horn, target complex and proton-beam absorber. The siting at CERN will be considered to allow preliminary investigations of the civil engineering to be carried out. An important aspect of the feasibility study will be consideration of the radiation-protection issues raised by the target and the pion and muon fluxes.

The potential of the cross-section-measurement programme was evaluted in [13] assuming a neutrino detector with the performance of the HiResM $v$ detector [61]. The precision with which the CCQE cross section could be measured is shown as a function of neutrino energy, $E_{v}$, in figure 8. A compilation of measurements of the CCQE cross section is also shown. The figure shows that nuSTORM has the potential to improve the systematic uncertainty on muon-neutrino (muon-antineutrino) CCQE cross section measurements by a factor of $\sim 5-6$. The $v_{e} A\left(\bar{v}_{e} A\right)$ cross-section measurements that can be made with nuSTORM will be unique.

The study of nuSTORM within the PBC Study Group will not include the neutrino detectors. Rather, examples of detectors that are under development to serve as near detectors for DUNE or Hyper-K will be considered as options. A physics study group has been set up to work in parallel with the accelerator study. The aim is that a publication describing the neutrino-scattering physics will be brought forward in the summer of 2018. The PBC study group mandate is to produce a final report by the end of 2018 [69].

\section{Conclusions}

Muon accelerators have the potential to serve as uniquely precise sources of electron and muon neutrino beams and to provide a route to multi-TeV lepton-anti-lepton collisions. The Neutrinos from Stored Muons (nuSTORM) facility is capable of delivering measurements of $v_{e, \mu} N\left(\bar{v}_{e, \mu} N\right)$ 

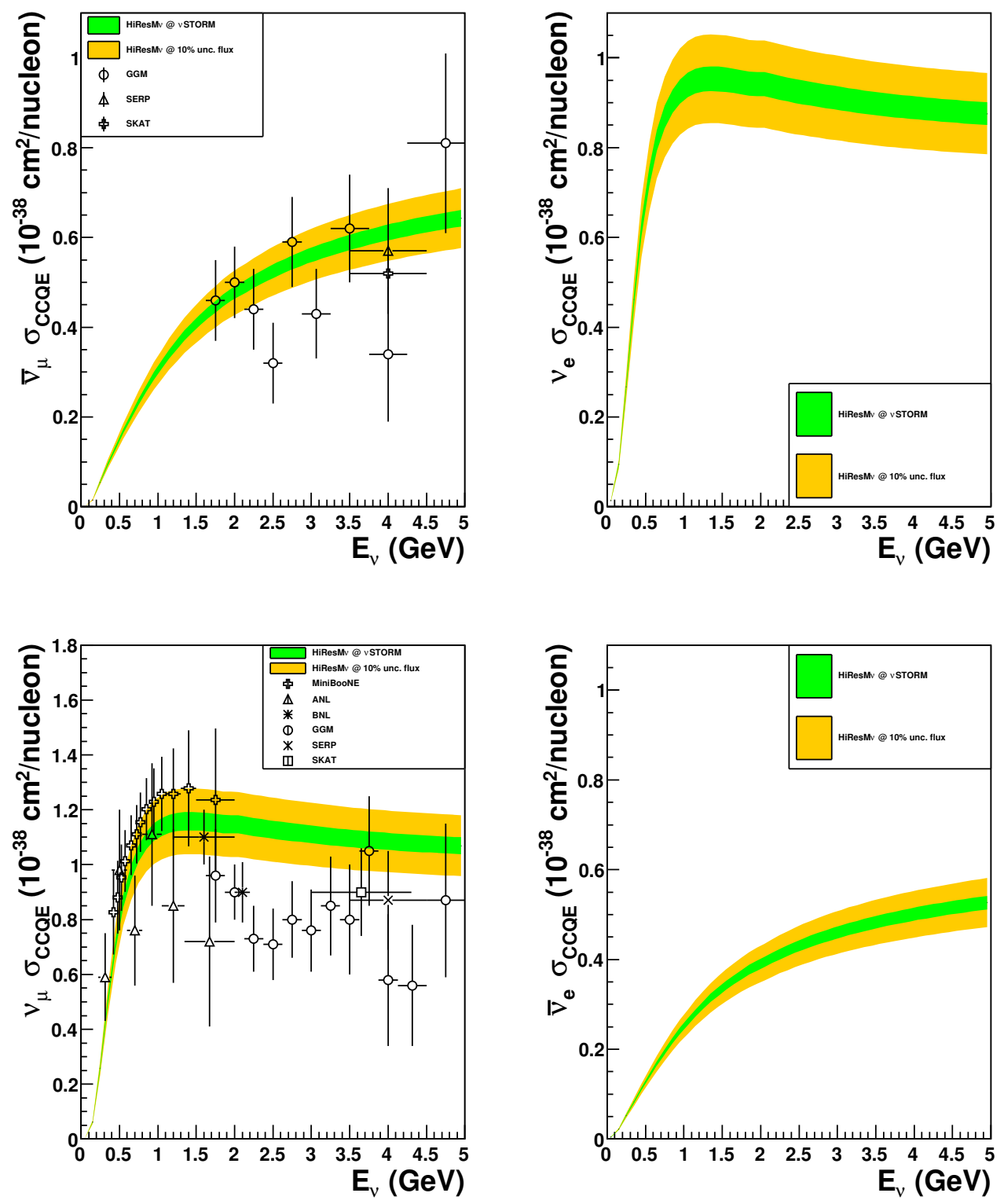

Figure 8: The CCQE cross section $\left(\sigma_{\mathrm{CCQE}}\right)$ plotted as a function of incident neutrino energy $\left(E_{v}\right)$ (taken from [13]). The cross sections that would be obtained with stored $\mu^{+}$beams are shown in the top row; the $\bar{v}_{\mu}$ CCQE cross secion is shown in the top left panel while the $v_{e}$ CCQE cross section is shown in the top right panel. The cross sections that would be obtained with stored $\mu^{-}$beams are shown in the bottom row; $v_{\mu}$ CCQE cross secion in the bottom left panel, $v_{e}$ CCQE cross section in the bottom right panel. The width of the coloured bands represent the systematic uncertainty on the cross sections determined using the HuResM $v$ detector [61] at the $v$ STORM facility. The green band shows the detector uncertainties combined with the $1 \%$ uncertainty on the neutrino flux at $v$ STORM. The yellow band shows the detector uncertainties combined with a flux uncertainty of $10 \%$. Measurements made by the MiniBooNE (solid diamonds, [62]), ANL (open triangles, [63]), BNL (crosses, [64, 65]), Gargamelle (open circles, [66]), SERP (stars, [67]) and SKAT (inverted open triangles, [68]) collaborations are also shown. 
scattering for which the flux uncertainty can be reduced to $1 \%$ or better. Such measurements will reduce the systematic uncertainties and biases in future long-baseline neutrino-oscillation experiments thereby enhancing their sensitivity to leptonic CP-invariance violation and improving the precision of their measurements of the oscillation parameters. In addition, accurate simulations of neutrino-nucleus scattering and precise calculations of nuclear matrix elements are required, for example, to: distinguish a signal for a light sterile-neutrino state from an effect arising from hadronisation or the nuclear inventory in power reactors; to interpret null results, or evaluate a discovery signal, in searches for neutrinoless double-beta decay; and to improve models of the evolution of core-collapse supernovæ.

The cross-section measurement programme at nuSTORM has the potential to contribute to the understanding of nuclear physics through the use of a pure weak probe that is $100 \%$ polarised. Should the results of the FNAL Short Baseline Neutrino Program indicate that future searches for light sterile neutrinos are required, nuSTORM can support an exquisitely sensitive sterile-neutrino search programme.

The implementation of nuSTORM at CERN is being studied within the Physics Beyond Colliders Study Group. The goals of the study are to provide a preliminary proposal for siting the facility at CERN, to re-optimise the facility for the neutrino-scattering programme and to demonstrate through simulation that the normalisation of the neutrino flux can be constrained to $\lesssim 1 \%$. The programme described here will establish nuSTORM as an option for CERN in time for the next update of the European Strategy for Particle Physics.

\section{Acknowledgements}

I would like to thank the organisers of the Neutrino telescopes workshops for the opportunity to describe the potential of nuSTORM and to outline with work being carried out in the CERN Physics Beyond Colliders Study Group. I acknowledge with gratitude the work of my many friends and colleagues in the nuSTORM, neutrino factory and MICE communities. In particular I would like to thank A. Bross, A. Liu, D. Neuffer and P. Huber on whose work I have drawn freely in preparing this contribution and J. Morfin for helpful comments on the manuscript.

\section{References}

[1] S. Geer, Neutrino beams from muon storage rings: Characteristics and physics potential, Phys. Rev. D57 (1998) 6989-6997, [hep-ph/9712290].

[2] V. Barger, S. Geer and K. Whisnant, Long baseline neutrino physics with a muon storage ring neutrino source, Phys. Rev. D 61 (2000) 053004, [9906487].

[3] D. Finley and N. Holtkamp, A feasibility study of a neutrino source based on a muon storage ring, Nucl. Instrum. Meth. A472 (2000) 388-394.

[4] S. Ozaki, R. B. Palmer, M. S. Zisman and J. C. Gallardo, Feasibility Study-II of a Muon-Based Neutrino Source, Tech. Rep. BNL-52623, Brookhaven National Laboratory, Upton, NY, 2001.

[5] ISS Physics Working Group collaboration, A. Bandyopadhyay et al., Physics at a future Neutrino Factory and super-beam facility, Rept. Prog. Phys. 72 (2009) 106201, [0710 . 4947]. 
[6] IDS-NF collaboration, S. Choubey et al., International Design Study for the Neutrino Factory, Interim Design Report, 1112.2853.

[7] ISS Detector Working GROUP collaboration, T. Abe et al., Detectors and flux instrumentation for future neutrino facilities, JINST 4 (2009) T05001, [0 712.4129].

[8] ISS ACCElerator Working Group collaboration, M. Apollonio et al., Accelerator design concept for future neutrino facilities, JINST 4 (2009) P07001, [0 802 . 4023 ].

[9] S. Geer, Muon colliders move nearer, CERN Cour. 37N10 (1997) 1-3.

[10] J. C. Gallardo et al., Muon Collider: Feasibility Study, 1996.

[11] J.-P. Delahaye et al., Enabling Intensity and Energy Frontier Science with a Muon Accelerator Facility in the U.S.: A White Paper Submitted to the 2013 U.S. Community Summer Study of the Division of Particles and Fields of the American Physical Society, 1308.0494.

[12] NUSTORM COLLABORATION collaboration, P. Kyberd et al., nuSTORM - Neutrinos from STORed Muons: Letter of Intent to the Fermilab Physics Advisory Committee, 1206.0294.

[13] NUSTORM collaboration, D. Adey et al., nuSTORM - Neutrinos from STORed Muons: Proposal to the Fermilab PAC, tech. rep., Fermi National Accelerator Laboratory, 2013.

[14] LSND collaboration, A. Aguilar-Arevalo et al., Evidence for neutrino oscillations from the observation of anti-neutrino(electron) appearance in a anti-neutrino(muon) beam, Phys. Rev. D64 (2001) 112007, [hep-ex/0104049].

[15] MiniBoonE collaboration, A. A. Aguilar-Arevalo et al., Unexplained Excess of Electron-Like Events From a 1-GeV Neutrino Beam, Phys. Rev. Lett. 102 (2009) 101802, [0812 . 2243 ].

[16] MiniBooNE collaboration, A. A. Aguilar-Arevalo et al., Improved Search for $\bar{v}_{\mu} \rightarrow \bar{v}_{e}$ Oscillations in the MiniBooNE Experiment, Phys. Rev. Lett. 110 (2013) 161801, [1207. 480 9].

[17] NUSTORM COLLABORATION collaboration, D. Adey et al., Light sterile neutrino sensitivity at the nuSTORM facility, Phys.Rev. D89 (2014) 071301, [1402.5250].

[18] Jaeckel, J. and Lamont. M. and Vallee, C., "Physics Beyond Colliders.” http://pbc.web.cern.ch, 2016.

[19] B. Pontecorvo, Inverse beta processes and nonconservation of lepton charge, Sov. Phys. JETP 7 (1958) 172-173.

[20] B. Pontecorvo, Neutrino experiments and the question of leptonic-charge conservation, Sov. Phys. JETP 26 (1968) 984-988.

[21] Z. Maki, M. Nakagawa and S. Sakata, Remarks on the unified model of elementary particles, Prog. Theor. Phys. 28 (1962) 870-880.

[22] Particle Data Group collaboration, C. Patrignani et al., Review of Particle Physics, Chin. Phys. C40 (2016) 100001.

[23] The T2K collaboration, “T2K.” http://t2k-experiment.org, 2013.

[24] Hyper-Kamiokande Working Group collaboration, K. Abe et al., A Long Baseline Neutrino Oscillation Experiment Using J-PARC Neutrino Beam and Hyper-Kamiokande, 1412.4673.

[25] Hyper-Kamiokande Proto-Collaboration collaboration, K. Abe et al., Physics potential of a long-baseline neutrino oscillation experiment using a J-PARC neutrino beam and Hyper-Kamiokande, PTEP 2015 (2015) 053C02, [1502 . 05199].

[26] HYPER-KAMIOKANDE collaboration, Hyper-Kamiokande Design Report, . 
[27] Hyper-Kamiokande Proto- collaboration, K. Abe et al., Physics Potentials with the Second Hyper-Kamiokande Detector in Korea, 1611.06118.

[28] The NOvA collaboration, "NOvA Neutrino Experiment.” https://www-nova.fnal.gov, 2016.

[29] DUNE collaboration, R. Acciarri et al., Long-Baseline Neutrino Facility (LBNF) and Deep Underground Neutrino Experiment (DUNE), 1601.05471.

[30] DUNE collaboration, R. Acciarri et al., Long-Baseline Neutrino Facility (LBNF) and Deep Underground Neutrino Experiment (DUNE), 1512.06148.

[31] DUNE collaboration, J. Strait et al., Long-Baseline Neutrino Facility (LBNF) and Deep Underground Neutrino Experiment (DUNE), 1601.05823.

[32] DUNE collaboration, R. Acciarri et al., Long-Baseline Neutrino Facility (LBNF) and Deep Underground Neutrino Experiment (DUNE), 1601.02984.

[33] A. M. Ankowski and C. Mariani, Systematic uncertainties in long-baseline neutrino-oscillation experiments, J. Phys. G44 (2017) 054001, [1609.00258].

[34] J. Cao et al., Roadmap for the international, accelerator-based neutrino programme, 1704.08181.

[35] P. Coloma and P. Huber, Impact of nuclear effects on the extraction of neutrino oscillation parameters, Phys. Rev. Lett. 111 (2013) 221802, [1307 . 1243].

[36] P. Coloma, P. Huber, C.-M. Jen and C. Mariani, Neutrino-nucleus interaction models and their impact on oscillation analyses, Phys. Rev. D89 (2014) 073015, [1311.4506].

[37] A. M. Ankowski, P. Coloma, P. Huber, C. Mariani and E. Vagnoni, Missing energy and the measurement of the CP-violating phase in neutrino oscillations, Phys. Rev. D92 (2015) 091301, [1507.08561].

[38] M. Ankowski, A., O. Benhar, P. Coloma, P. Huber, C. M. Jen, C. Mariani et al., Neutrino energy reconstruction in disappearance experiments with calorimetric and kinematic methods, Nuovo Cim. C39 (2016) 233.

[39] A. M. Ankowski, Nuclear effects are relevant to the calorimetric reconstruction of neutrino energy, 1704.07835.

[40] Y.-B. Yang, R. S. Sufian, A. Alexandru, T. Draper, M. J. Glatzmaier, K.-F. Liu et al., Glue Spin and Helicity in the Proton from Lattice QCD, Phys. Rev. Lett. 118 (2017) 102001, [1 609.05937 ].

[41] O. Hen, G. A. Miller, E. Piasetzky and L. B. Weinstein, Nucleon-Nucleon Correlations, Short-lived Excitations, and the Quarks Within, 1611.09748.

[42] O. Benhar, Modelling Neutrino-Nucleus Interactions: Status and Perspectives, JPS Conf. Proc. 12 (2016) 010001.

[43] L. Alvarez-Ruso et al., NuSTEC White Paper: Status and Challenges of Neutrino-Nucleus Scattering, 1706.03621.

[44] R. McKeown, Jefferson lab science opportunities at 12 gev, 2017.

[45] T. Lackowski, S. Dixon, R. Jedziniak, M. Blewitt and L. Fink, nuSTORM Project Definition Report, tech. rep., Fermi National Accelerator Laboratory, 2013.

[46] P. Kyberd et al., nustorm costing document, May, 2013. 
[47] D. Adey, C. Ankenbrandt, S. Agarwalla, R. Asfandiyarov, J. Back, G. Barker et al., Neutrinos from stored muons (storm): Expression of interest, Tech. Rep. CERN-SPSC-2013-015. SPSC-EOI-009, CERN, Geneva, Apr, 2013.

[48] D. Adey, S. Agarwalla, C. Ankenbrandt, R. Asfandiyarov, J. Back et al., Neutrinos from Stored Muons nuSTORM: Expression of Interest, 1305.1419.

[49] A. Liu, A. Bross and D. Neuffer, Optimization of the magnetic horn for the nuSTORM non-conventional neutrino beam using the genetic algorithm, Nucl. Instrum. Meth. A794 (2015) 200-205.

[50] A. Liu, D. Neuffer and A. Bross, Design and Simulation of the nuSTORM Pion Beamline, Nucl. Instrum. Meth. A801 (2015) 44-50.

[51] A. Liu, A. Bross and D. Neuffer, A FODO racetrack ring for nuSTORM: design and optimization, JINST 12 (2017) P07018, [1704.00798].

[52] R. Appleby, J. Garland, H. Owen, S. Tygier, K. Hock, J.-B. Lagrange et al., Dynamic Aperture Studies of the nuSTORM FFAG Ring, tech. rep., 2014.

[53] J.-B. Lagrange, R. Appleby, A. Bross, J. Garland, A. Liu, H. Owen et al., nuSTORM FFAG Decay Ring, 2016. 10.18429/JACoW-IPAC2016-THPMB053.

[54] D. Adey, R. Bayes, A. Bross and P. Snopok, nuSTORM and A Path to a Muon Collider, Ann. Rev. Nucl. Part. Sci. 65 (2015) 145-175.

[55] The Short Baseline Neutrino Program, “The Short Baseline Neutrino Program.” https://web.fnal.gov/collaboration/sbn/_layouts/15/start.aspx\#/SitePages/Home.aspx, 2014.

[56] D. Stratakis, Six-Dimensional Ionization Cooling for Muon Accelerators with Vacuum RF Technology, ICFA Beam Dyn. Newslett. 65 (2014) 54-62.

[57] The MICE collaboration, "INTERNATIONAL MUON IONIZATION COOLING EXPERIMENT." http://mice.iit.edu.

[58] J. A. Formaggio and G. P. Zeller, From eV to EeV: Neutrino Cross Sections Across Energy Scales, Rev. Mod. Phys. 84 (2012) 1307-1341, [1305. 7513].

[59] M. Wilking, E61: The j-parc intermediate water cherenkov detector, 2017.

[60] D. Casper, The Nuance neutrino physics simulation, and the future, Nucl. Phys. Proc. Suppl. 112 (2002) 161-170, [hep-ph/0208030].

[61] S. Mishra, Precision measurements and a high resolution near detector for Long-Baseline Neutrino program, Prog.Part.Nucl.Phys. 64 (2010) 202-204.

[62] MiniBoone Collaboration collaboration, A. Aguilar-Arevalo et al., Measurement of the Neutrino Neutral-Current Elastic Differential Cross Section on Mineral Oil at $E_{V} \sim 1$ GeV, Phys. Rev. D82 (2010) 092005, [1007.4730].

[63] W. A. Mann et al., Study of the reaction nu $n \rightarrow$ mu-p, Phys. Rev. Lett. 31 (1973) 844-847.

[64] G. Fanourakis, L. K. Resvanis, G. Grammatikakis, P. Tsilimigras, A. Vayaki, U. Camerini et al., Study of Low-energy Anti-neutrino Interactions on Protons, Phys. Rev. D21 (1980) 562.

[65] N. J. Baker, A. M. Cnops, P. L. Connolly, S. A. Kahn, H. G. Kirk, M. J. Murtagh et al., Quasielastic Neutrino Scattering: A Measurement of the Weak Nucleon Axial Vector Form-Factor, Phys. Rev. D23 (1981) 2499-2505. 
[66] S. Bonetti, G. Carnesecchi, D. Cavalli, P. Negri, A. Pullia, M. Rollier et al., Study of Quasielastic Reactions of Neutrino and anti-neutrino in Gargamelle, Nuovo Cim. A38 (1977) 260-270.

[67] S. V. Belikov et al., Quasielastic Neutrino and Anti-neutrinos Scattering: Total Cross-sections, Axial Vector Form-factor, Z. Phys. A320 (1985) 625.

[68] SKAT collaboration, J. Brunner et al., Quasielastic Nucleon and Hyperon Production by Neutrinos and Anti-neutrinos With Energies Below 30-GeV, Z. Phys. C45 (1990) 551.

[69] Jaeckel, J. and Lamont. M. and Vallee, C., "Mandate of the "Physics Beyond Colliders" Study Group.” http://pbc.web.cern.ch/doc/PCB-mandate.pdf, 2016. 